\title{
AN EXCESS DUE TO SMALL GRAINS AROUND THE NEARBY K0 V STAR HD 69830: ASTEROID OR COMETARY DEBRIS?
}

\author{
C. A. Beichman \\ Michelson Science Center, California Institute of Technology, M/S 100-22, Pasadena, CA 91125; chas@pop.jpl.nasa.gov \\ G. Bryden, T. N. Gautier, K. R. Stapelfeldt, and M. W. Werner \\ Jet Propulsion Laboratory, California Institute of Technology, 4800 Oak Grove Drive, Pasadena, CA 91109 \\ AND \\ K. Misselt, G. Rieke, J. Stansberry, and D. Trilling \\ Steward Observatory, University of Arizona, 933 North Cherry Avenue, Tucson, AZ 85721-0065 \\ Received 2005 January 7; accepted 2005 February 21
}

\begin{abstract}
Spitzer photometry and spectroscopy of the star HD 69830 reveal an excess of emission relative to the stellar photosphere between 8 and $35 \mu \mathrm{m}$ dominated by strong features attributable to crystalline silicates with an emitting surface area more than 1000 times that of our zodiacal cloud. The spectrum closely resembles that of the comet $\mathrm{C} / 1995 \mathrm{O} 1$ (Hale-Bopp). Since no excess is detected at $70 \mu \mathrm{m}$, the emitting material must be quite warm, be confined within a few $\mathrm{AU}$ of the star, and originate in grains with low, long-wavelength emissivity, i.e., grains much smaller than $70 \mu \mathrm{m} / 2 \pi \sim 10 \mu \mathrm{m}$. The strong mineralogical features are evidence for even smaller, possibly submicronsized grains. This small grain size is in direct contrast to the $10-100 \mu \mathrm{m}$ grains that dominate the relatively featureless spectra of our zodiacal dust cloud and most other main-sequence stars with excesses. The upper limit at $70 \mu \mathrm{m}$ also implies that any Kuiper Belt analog must be either very cold or less massive than $\sim 5$ times our own Kuiper Belt. With collisional and Poynting-Robertson drag times of less than $1000 \mathrm{yr}$ for small grains, the emitting material must either (1) be created through continual grinding down of material in a dense asteroid belt, or (2) originate in cometary debris arising from either a single "supercomet" or a very large number of individual comets arriving from a distant reservoir. In the case of a cometary origin for the emission, the mass requirements for continuous generation by many individual comets are unreasonable, and we favor the capture of a single super comet into a $0.5-1 \mathrm{AU}$ orbit, where it can evolve a large number of small grains over a 2 Myr period.
\end{abstract}

Subject headings: circumstellar matter — comets: general — infrared: stars — Kuiper Belt — planetary systems Online material: color figures

\section{INTRODUCTION}

The debris disk phenomenon, first discovered by the Infrared Astronomy Satellite (IRAS: Aumann et al. 1984; Gillett 1986; Backman \& Paresce 1993), is of great interest because of the clues these disks offer toward understanding the formation and evolution of planetary systems. Toward this end, aspects of debris disks have been studied with the Infrared Space Observatory (ISO: Habing et al. 2001; Decin et al. 2003), optical imaging (e.g., Smith \& Terrile 1984; Heap et al. 2000), and at submillimeter wavelengths (Holland et al. 1998; Greaves et al. 1998; Dent et al. 2000).

Information on the nature of the dust grains in these disks is particularly important. Spectroscopic observations toward luminous Herbig Ae/Be stars such as HD 100546 (Grady et al. 1997; Malfait et al. 1998; Meeus et al. 2001) and in young debris stars such as $\beta$ Pic (Telesco \& Knacke 1991) and $51 \mathrm{Oph}$ (Fajardo-Acosta et al. 1993) revealed dust composed, at least in part, of small (submicron) grains of crystalline silicates such as forsterite and enstatite. The similarity of these spectral features to those seen in comet C/1995 O1 (Hale-Bopp: Crovisier et al. 1996; Grün et al. 2001; Wooden et al. 2000) suggests that this circumstellar material may represent cometary debris. Yet these spectral features are not present in all or even most debris disks. More than a dozen classic debris disks (including Fomalhaut) examined by Spitzer (Jura et al. 2004; Stapelfeldt et al. 2004) show little or no spectral structure, suggesting that the grains in these systems are larger than $\sim 10 \mu \mathrm{m}$. These grains may be similar to those in our own zodiacal cloud, which are predominantly larger than $10-100 \mu \mathrm{m}$ with only a small admixture of smaller silicate grains yielding a weak $10 \mu \mathrm{m}$ emission feature (Kelsall et al. 1998; Fixsen \& Dwek 2002; Reach et al. 2003).

In this paper we report the detection of a large excess due to hot grains orbiting the star HD 69830. These grains are dominated by a population of crystalline silicates with prominent features in the Infrared Spectrograph (IRS) spectrum.

\section{PROPERTIES OF HD 69830}

HD 69830 (Gliese 302, BD-12 2449, HR 3259) is a bright $(V=5.95 \mathrm{mag})$, nearby $(12.6 \mathrm{pc})$, main-sequence dwarf of solar metallicity $[\mathrm{Fe} / \mathrm{H}]=0.015$ (Cayrel de Strobel et al. 2001). Its spectral type has been variously classified as between G8 (Skiff 2003) and K0 (Song et al. [2000] and other references in SIMBAD). We adopt a K0 V spectral type throughout this paper, which implies an effective temperature of $5150 \mathrm{~K}$, a mass of $0.8 M_{\odot}$, and a total luminosity of $0.45 L_{\odot}$ (Cox 2000). Using isochrone fitting, $\mathrm{Li}$ absorption, $\mathrm{Ca}$ II $\mathrm{H}$ and $\mathrm{K}$ line activity, weak $\mathrm{X}$-ray emission, and space motions, Song et al. (2000) suggested an age older than $0.6 \mathrm{Gyr}$ and up to $2 \mathrm{Gyr}$, with a preference for the older age. Examination of $\mathrm{Ca}$ II $\mathrm{H}$ and $\mathrm{K}$ activity indices 
TABLE 1

INFRARED PHOTOMETRY OF HD 69830

\begin{tabular}{ccccc}
\hline \hline Instrument & $\begin{array}{c}\lambda \\
(\mu \mathrm{m})\end{array}$ & \multicolumn{1}{c}{$\begin{array}{c}F_{\nu} \\
(\mathrm{Jy})\end{array}$} & $\begin{array}{c}\text { Photosphere }^{\mathrm{a}} \\
(\mathrm{Jy})\end{array}$ & Significance, $\chi^{\mathrm{b}}$ \\
\hline IRAS ................ & 12.0 & $0.68 \pm 0.041$ & 0.61 & 1.7 \\
MIPS ............ & 23.7 & $0.23 \pm 0.012$ & 0.16 & 6.1 \\
IRAS ................ & 25.0 & $0.24 \pm 0.026$ & 0.14 & 3.8 \\
MIPS ............ & 70.0 & $0.019 \pm 0.003$ & 0.018 & 0.4 \\
\hline
\end{tabular}

${ }^{\text {a }}$ From Kurucz model fitted to short-wavelength data.

b $\chi=($ Observed - Kurucz $) /\left[\text { noise }^{2}+(\text { model uncertainty })^{2}\right]^{1 / 2}$.

leads G. W. Marcy \& D. Fischer (2005, private communication) and Wright et al. (2004) to prefer an age of 2-4.7 Gyr, while IUE spectra suggest an age of 3 Gyr (Hufnagel \& Smith 1994). We adopt $2 \mathrm{Gyr}$ as the probable age for this star.

No planets have been detected orbiting HD 69830 using the radial velocity technique with a limit on any planet with a mass $M \sin (i)>0.5 M_{\mathrm{J}}$ located within a distance of $3 \mathrm{AU}$ of the star (G. W. Marcy \& D. Fischer 2005, private communication). Although Mannings \& Barlow (1998) noted the presence of a small "hot" excess based on IRAS observations at $25 \mu \mathrm{m}(3.8 \sigma$; Table 1), IRAS did not detect the star at either 60 or $100 \mu \mathrm{m}$. The star was not observed with $I S O$.

\section{SPITZER OBSERVATIONS}

\subsection{Observations and Data Reduction}

Spectra of HD 69830 were obtained with Spitzer on 2004 April 18 (AOR 4016640). These measurements were part of a program that has observed 36 main-sequence stars to look for disks (Beichman et al. 2005; G. Bryden et al. 2005, in preparation) using IRS (Houck et al. 2004). Standard IRS staring mode observations were made with the Short Low Order 1 (SL1; 7 $14 \mu \mathrm{m}$ ), Long Low Order 2 (LL2; 14-20 $\mu \mathrm{m}$ ), and Long Low Order 1 (LL1; 20-35 $\mu \mathrm{m}$ ) modules. Each star was observed at two positions along the slit, called $\operatorname{Nod}_{1}$ and $\operatorname{Nod}_{2}$. Data were processed by the Spitzer Science Center (SSC) to produce calibrated images of the spectrometer focal plane. The SSC SPICE software was used to extract spectra from these images. Because of the need for careful subtraction of the stellar continuum to detect a faint excess, additional steps were taken in producing the final spectra:

1. To ensure proper sky subtraction, differences were formed between the two Nod positions to produce two sky-subtracted images $\left(\operatorname{Diff}_{1}=\operatorname{Nod}_{1}-\mathrm{Nod}_{2}\right.$ and Diff $\left.2=\operatorname{Nod}_{2}-\operatorname{Nod}_{1}\right)$. SPICE was used to extract one-dimensional spectra from each difference image using default settings and calibration.

2. The first few points at the beginning and end of the spectrum from each module were typically unreliable, as were a few bad pixels flagged in the SSC processing. These were rejected. This effect was particularly noticeable at the long-wavelength end of SL1 $(\lambda>14 \mu \mathrm{m})$. Since the short-wavelength end of LL2 overlaps SL1, there is no gap in the final spectrum.

3. Longward of $14 \mu \mathrm{m}$, photospheres of solar-type stars are smooth and do not differ greatly from a Rayleigh-Jeans blackbody (Kurucz 1992; Castelli \& Kurucz 2003). We used this fact to improve the pixel-to-pixel calibration in the extracted SSC SPICE spectra; 35 of the 36 stars observed with IRS as part of this program showed no evidence for an excess at either 24 or $70 \mu \mathrm{m}$ with MIPS but did show consistent pixel-to-pixel deviations from a smooth photospheric model. From these stars we formed ratios of the extracted IRS spectra to the Kurucz model appropriate for the effective temperature and metallicity of each star. From the average of these ratios at each wavelength, we created a "superflat" response curve for the SL1, LL2, and LL1 modules. Because of possible variability in the flat response in the SL1 module, we used only the eight other stars observed during the same instrument campaign (2004 April) as HD 69830 to generate the superflat for this module. For the LL modules we used data from all 35 stars. HD 69830 was excluded from the creation of the superflats.

The corrections derived from the comparison of extracted spectra and the photospheric models for the three modules are small:

1. Shortward of $14 \mu \mathrm{m}$, the SL1 superflat typically has values in the range $0.97-1.03$ or, equivalently, deviations from unity response of $\sim 3 \%$; a few pixels have values deviating from unity by $10 \%$. The values at each pixel of the SL1 superflat have a dispersion around the pixel response (averaged over all eight stars) of $\sigma_{\text {pop }} \sim 2 \%$ and $\sigma_{\text {mean }} \sim 0.4 \%$. In the LL1 and LL2 modules, the typical superflat values are in the range $0.98-1.02$, with dispersions (averaged over all 35 stars) of $\sigma_{\text {pop }} \sim 1 \%-2 \%$ and $\sigma_{\text {mean }} \sim 0.2 \%-0.3 \%$. We divided the extracted spectra by the appropriate superflat to remove any residual calibration variations. The dispersion in the superflat $\left(\sigma_{\mathrm{pop}}\right)$ is an indication of the limiting systematic noise in removing the photospheric contribution to the signal from these stars.

2. It was necessary to adjust the absolute flux scale of all of the IRS SSC spectra by a factor of $1.20 \pm 0.01$ to fit the photospheric models obtained by fits to shorter wavelength $(0.4-2.2 \mu \mathrm{m})$ data. With this adjustment, the SL1 data are consistent with the IRAS $12 \mu \mathrm{m}$ data and the LL2 data are consistent with the IRAS MIPS data at $24 \mu \mathrm{m}$. The origin of this absolute calibration factor, e.g., pointing errors or an imperfect aperture correction, is being pursued but is not relevant to the present investigation.

\subsection{Results}

The overall spectral energy distribution (SED) of HD 69830 including photometry (Table 1) and the IRS spectra is shown in Figure 1. A prominent excess is obvious in all three IRS modules, reaching $60 \%$ over the photospheric continuum at $35 \mu \mathrm{m}$.

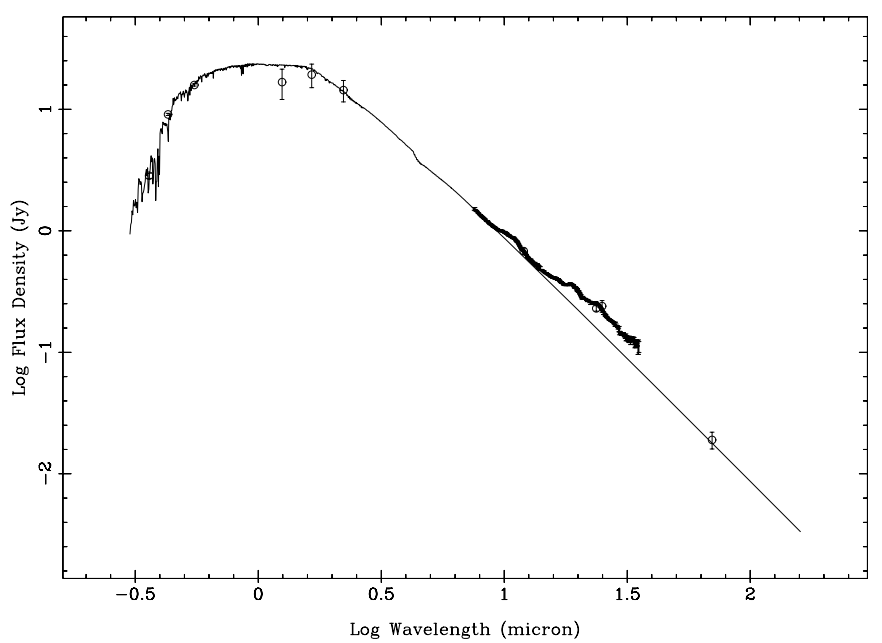

FIG. 1.-SED of HD 69830 shows a Kurucz photospheric model (solid line) fitted to short-wavelength data from Hipparcos and 2MASS (error bars). The 2MASS data $\left(J, H\right.$, and $\left.K_{s}\right)$ are quite uncertain because of the brightness of the star. Corrected spectra from the three IRS modules (thick line with error bars) are shown along with MIPS and IRAS photometry. [See the electronic edition of the Journal for a color version of this figure.] 


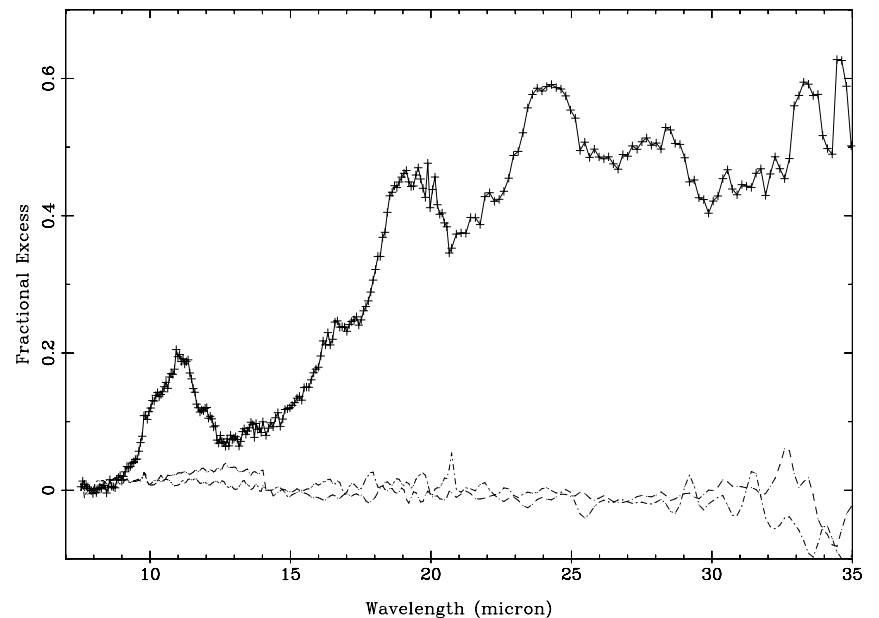

FIG. 2.-After correction with the "superflat," small deviations from a smooth photosphere are readily detectable in these IRS spectra. Shown are the fractional excesses, $\left(F_{\nu \text {, Obs }}-F_{\nu \text {, Kurucz }}\right) / F_{\nu \text {, Kurucz }}$, for two stars with no evidence for excess at any wavelength, HD 173667 (gray dotted line) and HD 142373 (gray dash-dotted line), and for HD 69830 (solid line), which shows prominent excesses from 8 to $35 \mu \mathrm{m}$. [See the electronic edition of the Journal for a color version of this figure.]

A number of broad spectral features, discussed below, are prominent in the data. Figure 2 compares the fractional deviation from a smooth photosphere for HD 69830 with the spectra of two stars, HD 173667 (AOR 4024320) and HD 142373 (AOR 4023040), that show no excesses at either IRAS or MIPS wavelengths. As is clear from this figure, the size and spectral content of HD 60930's excess are large compared to the uncertainties and will not be qualitatively altered by further refinements in the spectral extraction. Figure 2 can also be taken as a limit to the deviations that might be found toward any star, approximately $2 \%-3 \%$.

Included in Figure 1 and presented in Table 1 is MIPS photometry (AOR 4041728) as well as color-corrected IRAS data from the Faint Source Catalog (Moshir et al. 1990). The MIPS data were reduced using the MIPS GTO DAT software Gordon et al. 2005) and show a prominent (50\%) excess at $24 \mu \mathrm{m}$, confirming the excess hinted at in the IRAS data at $25 \mu \mathrm{m}$ (Mannings \& Barlow 1998). There is no evidence for extended emission in the $24 \mu \mathrm{m}$ image. The consistency of the IRAS and Spitzer MIPS data at 24 and $25 \mu \mathrm{m}$ suggests that there has been no strong variation on the $20 \mathrm{yr}$ timescale.

As discussed elsewhere, HD 69830 is the only star out of a sample of 84 showing a significant $24 \mu \mathrm{m}$ excess (Beichman et al. 2005; G. Bryden et al. 2005, in preparation). The uniqueness of HD 69830 in this sample, one out of 84 , or $1.2 \%$, confirms the rarity of hot excesses around mature FGK stars first observed by IRAS (Fajardo-Acosta et al. 2000) and ISO (Laureijs et al. 2002). But HD 69830 is even more exceptional in that its $24 \mu \mathrm{m}$ excess is not accompanied by a longer wavelength excess, as is normally the case for debris disk sources (Mannings \& Barlow 1998; Backman 1998). The measured value of the HD 69830 excess at $70 \mu \mathrm{m}$ is $1 \pm 3 \mathrm{mJy}$, compared to the predicted photospheric level of $18 \mathrm{mJy}$, or $F_{\text {excess }} / F_{\text {star }}<$ $50 \%$ (3 times the $1 \sigma$ statistical uncertainty combined in quadrature with a $20 \%$ overall calibration uncertainty) at $70 \mu \mathrm{m}$. The lack of a $70 \mu \mathrm{m}$ excess is evidence for the presence of small grains with low, far-infrared emissivity.

The top panel of Figure 3 shows the IRS data with the photospheric component subtracted to reveal the details of excess. Uncertainties at each wavelength were derived using the largest
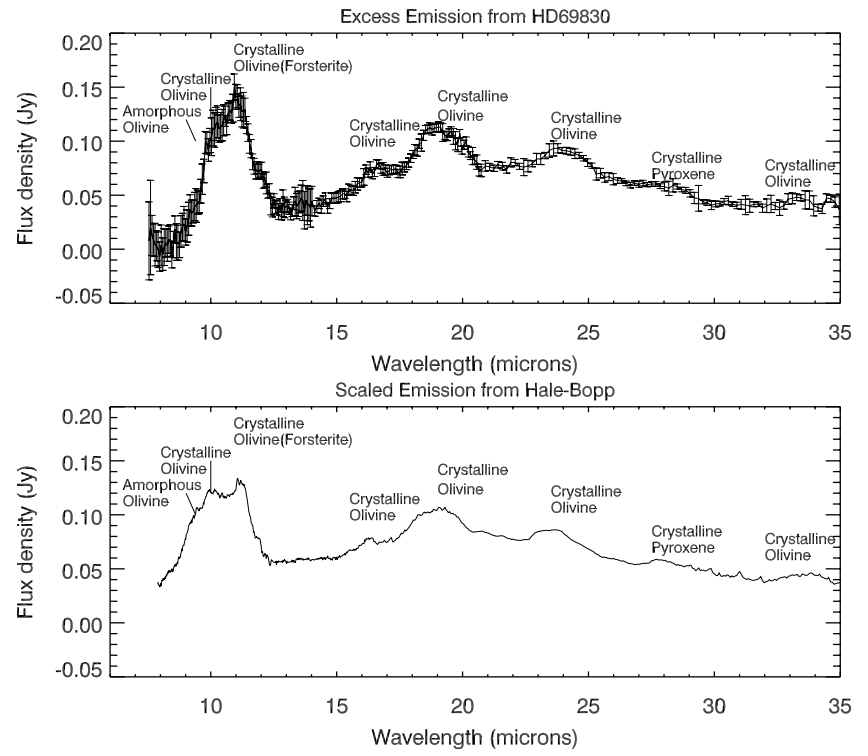

FIG. 3.-Top: Spectrum of the excess of HD 69830. Bottom: For comparison, the spectrum of the comet Hale-Bopp (Crovisier et al. 1996) normalized to $400 \mathrm{~K}$, as described in the text. Identifications of some of the mineralogical features (Table 2) are indicated.

of two terms: (1) the quoted errors in the individual SPICE extractions and (2) the difference between the corrected spectra obtained at the two different nod positions. To give the total uncertainty, the preceding value is combined in quadrature with a systematic term equal to $1 \%$ of the photospheric emission to account for errors in the subtraction of the photosphere. The first two terms reflect statistical uncertainties in the spectrum and become worse at the longest wavelengths where the signalto-noise ratio $(\mathrm{S} / \mathrm{N})$ is poorest. The third term, being proportional to the stellar brightness, decreases in importance at longer wavelengths.

\section{THE EXCESS TOWARD HD 69830}

The bottom panel of Figure 3 shows a spectrum obtained by ISO of the comet Hale-Bopp (Crovisier et al. 1996) and scaled as described in $\S 4.2$ to match the overall slope of the Spitzer data. The detailed similarity between the two spectra is remarkable and will be used as a guide to the identification of features in the spectrum of HD 69830.

\subsection{Features in the Spectrum of the Excess}

Table 2 identifies some of the features in the excess. Most prominent are broad features at 9.4-12, 16, 19.3, and $23.8 \mu \mathrm{m}$. Other features of moderate strength include a plateau at $10 \mu \mathrm{m}$, a plateau at $27 \mu \mathrm{m}$, and a weak feature of low $\mathrm{S} / \mathrm{N}$ around 33$34 \mu \mathrm{m}$. As noted in Table 2 and Figure 3, small Mg-rich crystalline silicate grains, ${ }^{1}$ e.g., enstatite and forsterite, have spectral features that match those in the spectrum remarkably well (Jäger et al. 1998; Koike et al. 1993). These are seen in the ISO and ground-based spectra of Hale-Bopp (Crovisier et al. 1996, 1997; Wooden et al. 1999, 2000). While not dominant in the spectrum of the zodiacal cloud, these crystalline silicates are found in interplanetary dust particles as well as in meteorite inclusions (Yoneda et al. 1993; Bradley 2003).

1 As discussed in Wooden et al. (1999, 2000), olivines are minerals with $\left[\mathrm{Mg}_{y} \mathrm{Fe}_{1-y}\right]_{2} \mathrm{SiO}_{4}$ and $0 \leq y \leq 1$; forsterite is an $\mathrm{Mg}$ pure olivine with $y=0$. Pyroxenes are minerals with $\left[\mathrm{Mg}_{x} \mathrm{Fe}_{1-x}\right] \mathrm{SiO}_{3}$ and $0 \leq x \leq 1$; enstatite is a $\mathrm{Mg}$ pure pyroxene with $x=0$. 
TABLE 2

Features in Excess Compared with Identifications in Comet Hale-Bopp

\begin{tabular}{|c|c|c|}
\hline $\begin{array}{c}\lambda \\
(\mu \mathrm{m})\end{array}$ & Comment & Identification \\
\hline $11(9.4-12.0) \ldots \ldots \ldots \ldots \ldots \ldots \ldots \ldots . .$. & Very strong emission & Crystalline forsterite \\
\hline 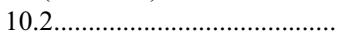 & Shoulder within $11 \mu \mathrm{m}$ feature & Crystalline olivine \\
\hline $11.0-11.3 \ldots \ldots \ldots \ldots \ldots \ldots \ldots \ldots \ldots . .$. & Peak within $11 \mu \mathrm{m}$ feature & Crystalline olivine \\
\hline 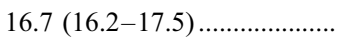 & Broad feature on shoulder of $19 \mu \mathrm{m}$ feature & Crystalline olivine \\
\hline $19.3(17.7-20.7) \ldots \ldots \ldots \ldots \ldots \ldots \ldots$ & Very broad emission feature & Crystalline olivine \\
\hline 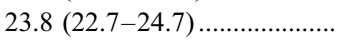 & Prominent, broad feature & Crystalline olivine \\
\hline 27.8 & Weak feature or plateau & Crystalline pyroxene (enstatite) \\
\hline $33-35$ & Weak feature & Crystalline olivine, low $\mathrm{S} / \mathrm{N}$ \\
\hline
\end{tabular}

Note.-From Crovisier et al. (1996, 1997), Wooden et al. (2000), and Henning (1998).

A detailed examination of the spectrum reveals that forsterite matches the 9-11 $\mu \mathrm{m}$ feature, while enstatite more closely matches the ratio of the 19 and $23.8 \mu \mathrm{m}$ features (Henning 1998). Features due to crystalline olivines are far more prominent than those due to pyroxenes, although an inflection point around $27 \mu \mathrm{m}$ might be due to crystalline pyroxene. The $9.3 \mu \mathrm{m}$ feature characteristic of small, amorphous silicate grains that is prominent in Hale-Bopp and seen weakly in our own zodiacal cloud (Reach et al. 2003) is not seen at all in HD 69830. Since the emission between the features does not drop to zero, there must be some source of continuum opacity, possibly larger grains, in both HD 69830 and Hale-Bopp. In summary, we attribute the excess toward HD 69830 to a mixture of grains similar to those seen in Hale-Bopp and very different from those responsible for the relatively featureless spectra of typical debris disks.

\subsection{Model for the Excess Emission}

The simplest model for the emission (Fig. 3) scales the ISO spectrum of Hale-Bopp by the ratio of two blackbodies,

$F_{\nu}($ HD 69830$) \propto F_{\nu}($ Hale-Bopp $) B_{\nu}(400 \mathrm{~K}) / B_{\nu}[T($ Hale-Bopp $)]$,

where $B_{\nu}(T)$ is the Planck function and $T$ (Hale-Bopp) $=207 \mathrm{~K}$ is the equilibrium temperature of a small crystalline silicate grain 2.9 AU from the Sun. The value of $400 \mathrm{~K}$ is fitted by eye to provide a reasonable fit to the HD 69830 excess and corresponds to an equilibrium distance of $\sim 0.5 \mathrm{AU}$ for small grains. Although the single temperature scaling gives a remarkably good representation of the data, we next develop a more realistic model that constrains the location, size, and composition of the emitting material.

The presence of pronounced spectral features on top of a weak continuum suggests optically thin emission dominated by dust grains comparable to or smaller than the wavelength: $a \leq$ $\lambda_{c} / 2 \pi \sim 1 \mu \mathrm{m}$, where $\lambda_{c} \sim 8 \mu \mathrm{m}$ is the shortest wavelength showing spectral features. The close analogy to Hale-Bopp requires the existence of grains as small as $0.25 \mu \mathrm{m}$ (Wooden et al. 1999, 2000). While fluffy, low-density grains with a size around $1 \mu \mathrm{m}$ have been suggested to fit the spectrum of HaleBopp (Li \& Greenberg 1998), for this preliminary analysis we adopt the following empirical model:

1. Synthesize empirical absorption cross sections, $Q_{\mathrm{abs}}$, derived from the scaled Hale-Bopp observations (Wooden et al. 1999, 2000), $Q_{\mathrm{abs}} \propto F_{\nu} / B_{\nu}\left(T_{\mathrm{HB}}\right)$, with $T_{\mathrm{HB}}=207 \mathrm{~K}$ and scaled to match laboratory measurements of crystalline olivine at $11.3 \mu \mathrm{m}$ (Mukai \& Koike 1990).
2. Subtract optical constants for $0.25 \mu \mathrm{m}$ amorphous silicate grains (Draine \& Lee 1984; Weingartner \& Draine 2001) from the optical constants derived from Hale-Bopp, since the $9.3 \mu \mathrm{m}$ feature produced by this type of grain is not seen in HD 69830. Examination of Hale-Bopp emission models, e.g., Figure 6 in Wooden et al. (1999) suggests that amorphous grains are at least a factor of 3 less abundant in HD 69830 than in Hale-Bopp.

3. Synthesize empirical absorption cross sections at $70 \mu \mathrm{m}$ as described above using the $70 \mu \mathrm{m}$ data for Hale-Bopp from Grün et al. (2001). The assumption that all the emission comes from grains following the same equilibrium temperature law is naive but gives a self-consistent estimate of the $70 \mu \mathrm{m}$ emission.

4. Assuming a flat-disk geometry for convenience, calculate the temperature gradient through radiative equilibrium of $0.25 \mu \mathrm{m}$ grains orbiting a $\mathrm{K} 0$ star and integrate the surface brightness profile, $I_{\nu}(r)=\tau(r) B_{\nu}[T(r)]$, over a region extending from the grain sublimation distance (at which $T \sim 1500 \mathrm{~K}$ and corresponding to $r \sim 0.06 \mathrm{AU}$ ) out to an outer radius $r_{\max }$.

5. Let the radial dependence of the optical depth follow a power-law distribution, $\tau(r) \propto r^{\alpha}$. For comparison, $\alpha=-0.4$ in our solar system.

A $\chi^{2}$ search was made through a broad range of values for the free parameters in the model: the surface area of the small grains derived from the Hale-Bopp spectrum, the power-law index of the surface density profile, and $r_{\max }$. The parameters of the best-fit model are given in Table 3. As shown in Figure 4, the model reproduces many, but not all, of the details in the IRS spectrum. Some problems include a shoulder at $10 \mu \mathrm{m}$ that

TABLE 3

Properties of Best-Fit Model

\begin{tabular}{|c|c|}
\hline Parameter & Model \\
\hline \multicolumn{2}{|l|}{ Best-Fit Model Parameters } \\
\hline 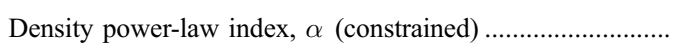 & -0.4 \\
\hline$r_{\max }(\mathrm{AU})$ & 1.0 \\
\hline 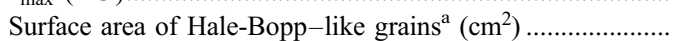 & $2.7 \times 10^{23}$ \\
\hline Reduced $\chi^{2}(250$ dof $)$ & 3.8 \\
\hline
\end{tabular}

Derived Parameters

Predicted $70 \mu \mathrm{m}$ flux density (mJy)... 0.75

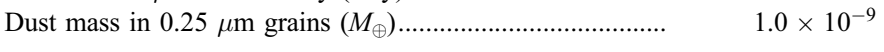

${ }^{\text {a }}$ Assumes optical constants derived from scaled Hale-Bopp spectrum with amorphous silicates subtracted to remove the $9.3 \mu \mathrm{m}$ feature seen in Hale Bopp but not in HD 69830. 


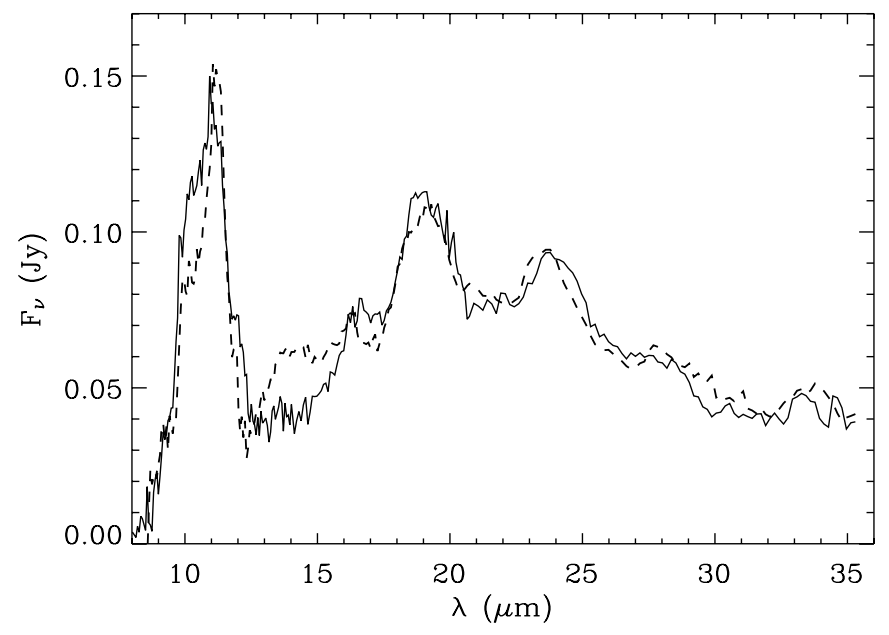

FIG. 4.-As described in the text, a simple model using crystalline silicate grains in a disk extending to $\sim 1 \mathrm{AU}$ (dashed line) is fitted to the IRS spectrum of the excess (solid line). [See the electronic edition of the Journal for a color version of this figure.]

cannot be fitted without throwing off the fit at $11 \mu \mathrm{m}$; an excess between 13 and $15 \mu \mathrm{m}$ not seen in Hale-Bopp (the feature does not correspond to known $\mathrm{Mg}$ - or $\mathrm{Mg} / \mathrm{Fe}$-rich silicates but resembles unidentified features in the spectrum of R Cas [Jäger et al. 1998]); and subtle variations around 19 and $24 \mu \mathrm{m}$, where four or five separate bands of forsterite and enstatite make up a broad feature sensitive to small composition and/ or temperature differences (Bowey et al. 2001). Despite these small but significant differences in detail, the Hale-Bopp based model provides an excellent analog for the HD 69830 debris disk.

In the final fit (Table 3 and Fig. 4) we constrained the powerlaw index of the radial surface density power law to have a value of -0.4 , since varying this parameter away from -0.4 did not significantly improve the overall results. Models with steeper power-law indices and $r_{\text {max }} \geq 5 \mathrm{AU}$ were consistent with the limit to the $70 \mu \mathrm{m}$ excess but had poor fits in the 7-35 $\mu \mathrm{m}$ region with a reduced $\chi^{2} \geq 10$. Models with $\alpha=-0.4$ and $r_{\max }>1$ AU also had poor $\chi^{2}$ values and were inconsistent with the $70 \mu \mathrm{m}$ limit. Although grain size was not an explicit free parameter in the model because we adopted opacities directly from the Hale-Bopp spectrum, we found that grains larger than about $5 \mu \mathrm{m}$ were inconsistent with the limits on the $70 \mu \mathrm{m}$ excess for any reasonable combination of model parameters.

The fractional luminosity in the excess, integrated between 7 and $35 \mu \mathrm{m}$, is $L_{\text {dust }} / L_{*}=2 \times 10^{-4}$, making this system a factor of 2 brighter than Fomalhaut ( $\alpha$ PsA: Backman \& Paresce 1993; Stapelfeldt et al. 2004) in terms of fractional dust luminosity and more than 1000 times brighter than our own zodiacal cloud. The surface area of emitting material in the HD 69830 cloud is $1.3 \times 10^{3}$ times that of our own zodiacal cloud, $\sim 2 \times$ $10^{20} \mathrm{~cm}^{2}$ within 2-3 AU (Backman 1998). In terms of its fractional surface area of emitting material, our solar zodiacal cloud has $\Sigma_{\text {frac }} \sim 1 \times 10^{-7}$, which compares to the fractional surface area of $0.25 \mu \mathrm{m}$ grains within 1 AU of $\Sigma_{\text {frac }} \sim 3 \times 10^{-4}$ for HD 69830.

If the emitting grains all have radius $a=0.25 \mu \mathrm{m}$ with density $\rho=3 \mathrm{~g} \mathrm{~cm}^{-3}$, then the mass of the emitting material (Table 3 ) would be $(4 / 3) \Sigma \rho a=2.7 \times 10^{19}$ g or $4.6 \times 10^{-9} M_{\oplus}$, where $\Sigma=$ $N_{\text {tot }} \pi a^{2}$ is the emitting surface area of $N_{\text {tot }}$ grains. However, since HD 69830 is probably surrounded by a broad distribution of grain sizes, the total mass could be much larger. For a grain size distribution $n(a)=n_{0}\left(a / a_{0}\right)^{-3.5}$, corresponding to predictions for a collisional cascade (Dohnanyi 1969), the ratio of total mass $M$ to surface area $\Sigma$ is $(M / \Sigma) \sim(4 / 3) \rho\left(a_{\min } a_{\max }\right)^{1 / 2}$, where $a_{\min }$ is the minimum grain size and $a_{\max }$ is the maximum grain size. For $a_{\min }=0.25 \mu \mathrm{m}$ and $a_{\max }=10 \mathrm{~km}$, the total mass of material associated with the disk emission (Table 3 ) is $5.4 \times 10^{24} \mathrm{~g}$, or $0.9 \times 10^{-3} M_{\oplus}$. Given the strong dependence on the unknown grain size distribution, this extrapolation is obviously very uncertain, e.g., integrating up to $100 \mathrm{~km}$ sized bodies would yield masses a factor of 3 larger. This number should be compared to the cumulative mass of asteroids up to $10 \mathrm{~km}$ in our solar system, $0.014 \times 10^{-3} M_{\oplus}$ (Bidstrup et al. 2005; Krasinsky et al. 2002), indicating a factor of 64 times more mass in a putative "asteroid" belt around HD 69830 than in our own asteroid belt (up to $10 \mathrm{~km}$ size). We analyze this estimate in the context of grain production and loss mechanisms in $\S 4.4$.

\subsection{Limits on a Kuiper Belt}

One of the most striking aspects of HD 69830 is the lack of a long-wavelength excess to accompany the $8-35 \mu \mathrm{m}$ excess. Treating the upper limit at $70 \mu \mathrm{m}$ just like the upper limits toward other main-sequence stars observed by MIPS (Beichman et al. 2005; G. Bryden et al. 2005, in preparation) yields an estimate for the amount of cold dust. The peak of the cold dust emission comes at $70 \mu \mathrm{m}$ for material at a temperature of $50 \mathrm{~K}$ located at $\sim 50 \mathrm{AU}$ from the star (for small grains; perhaps a factor of 2 closer in for larger grains). In this case, $L_{\text {dust }} / L_{*}<10^{-5}\left(5600 \mathrm{~K} / T_{*}\right)^{3}\left(F_{\text {dust }} / F_{*}\right) \sim 5 \times 10^{-6}(3 \sigma)$. This is only 5 times larger than the upper end of the range of values, $10^{-6}$ to $10^{-7}$, predicted for the Kuiper Belt region beyond $30 \mathrm{AU}$ in our solar system (Stern 1996) and less than the IRAS constraint, $<10^{-5}$ (Aumann \& Good 1990). The HD 69830 limit on cold material is uncertain, as it is based on only a single wavelength; observations at submillimeter wavelengths would further constrain the potential reservoir of distant cold material (Greaves et al. 2004).

\subsection{Origin of the Emitting Grains}

Nothing in the properties of HD 69830 suggests that this star is anything other than a mature, main-sequence dwarf. Thus, there is no reason to attribute its excess to dust associated with the weak mass loss often seen toward $\mathrm{M}$ and $\mathrm{K}$ giants (Beichman et al. 1990). Spectral features in the excess emission around such stars have been identified with small crystalline grains condensing in the quiescent, outflowing gas (Henning 1998; Dijkstra et al. 2003), but since HD 69830 is a quintessential dwarf star, we do not consider this possibility further. In the absence of any purely stellar phenomenon, we attribute the excess to orbiting dust derived from the solid material left over from the formation of the star itself, i.e., a debris disk. We now investigate the source of this dust in more detail.

\subsubsection{Timescales}

The rarity of main-sequence stars with hot excesses and without an associated colder debris cloud $(\leq 1 \%)$ suggests that the conditions for this excess are found around fewer than one star in 100 , or that it is a transitory (or low duty cycle) event lasting no more than $1 \%$ of the 2 Gyr lifetime of HD 69830 .

What are the natural timescales of the debris clouds in HD 69830 and, for comparison, in our solar system? A debris 
TABLE 4

Comparison of Solar System and HD 69830 Model

\begin{tabular}{|c|c|c|}
\hline Parameter & Solar System & HD 69830 \\
\hline \multicolumn{3}{|c|}{ System Parameters (see text) } \\
\hline Orbital distance $(\mathrm{AU})$ & 3 & 1 \\
\hline Stellar mass $\left(M_{\odot}\right)$ & 1 & 0.8 \\
\hline Stellar luminosity $\left(L_{\odot}\right)$ & 1 & 0.45 \\
\hline ............................. & $10-30$ & 0.25 \\
\hline 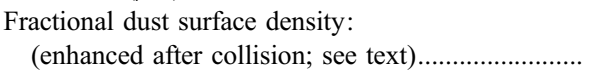 & $\begin{aligned} & 10^{-7}(<3 \mathrm{AU}) \\
& 6 \times 10^{-7}\end{aligned}$ & $\begin{array}{c}3 \times 10^{-4}(<1 \mathrm{AU}) \\
\ldots\end{array}$ \\
\hline 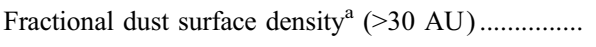 & $10^{-6}$ to $10^{-7}$ (estimate) & $<6 \times 10^{-6}$ \\
\hline \multicolumn{3}{|c|}{ Timescales for Dust Destruction (1 AU) } \\
\hline$\tau_{\text {coll }}(\mathrm{yr}) \ldots \ldots \ldots \ldots \ldots$ & $1 \times 10^{6}$ & 400 \\
\hline (n) & $(2-6) \times 10^{5}$ & 700 \\
\hline \multicolumn{3}{|c|}{ Asteroid Belt Mass $\left(a_{\max }=10 \mathrm{~km} ; M_{\oplus}\right)$} \\
\hline Our solar system ${ }^{\mathrm{b}}$. & $0.014 \times 10^{-3}$ & \\
\hline From emitting surface area ${ }^{c} .$. & $\ldots$ & $0.9 \times 10^{-3}$ \\
\hline 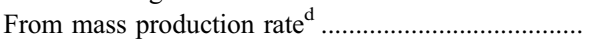 & $\ldots$ & $0.3 \times 10^{-3}$ \\
\hline From integrating mass-loss rate over 2 Gyr ................ & $\ldots$ & $(5-10) \times 10^{-3}$ \\
\hline
\end{tabular}

\footnotetext{
${ }^{\mathrm{a}}$ Stern (1996) for outer solar system and from $70 \mu \mathrm{m}$ limit for HD 69830.

b From Bidstrup et al. (2005) and Krasinsky et al. (2002).

c From scaling the emitting surface area to total mass (§ 4.2).

d Based on asteroid collisions for PR drag-dominated dust, $n_{\mathrm{gr}} \propto N_{c}^{2}$ (§ 4.4.2).
}

cloud continually loses material because of collisional grinding down of grains to a size small enough that radiation pressure and/or Poynting-Robertson (PR) drag ultimately removes them from the system. The relevant timescales (Burns et al. 1979; Backman \& Paresce 1993) are

$$
\tau_{\text {coll }}=\frac{t_{\text {orbit }}}{8 \Sigma_{\text {frac }}}=\frac{r_{\mathrm{AU}}^{1.5}}{8 \Sigma_{\text {frac }} M_{*}^{0.5}} \mathrm{yr},
$$

where $\Sigma_{\text {frac }}$ is the fractional surface area of the dust (Backman $\&$ Paresce 1993), and the PR drag time (Backman \& Paresce 1993),

$$
\tau_{\mathrm{PR}}=700 a_{\mathrm{gr}} \rho r_{\mathrm{AU}}^{2} \frac{L_{\odot}}{L_{*}} \mathrm{yr}
$$

where $\rho\left(\mathrm{g} \mathrm{cm}^{-3}\right)$ is the particle density and the grain size is in $\mu \mathrm{m}$.

Table 4 compares relevant parameters for our solar system's zodiacal cloud and that of HD 69830. In our solar system the critical (shortest) timescale is the PR time, $\tau_{\mathrm{PR}}=(2-6) \times 10^{5} \mathrm{yr}$ for 10-30 $\mu \mathrm{m}$ grains (Fixsen \& Dwek 2002; Reach et al. 2003). In HD 69830 the collisional timescale is $400 \mathrm{yr}$ and the PR timescale is about $700 \mathrm{yr}$ for $0.25 \mu \mathrm{m}$ grains at $1 \mathrm{AU}$. The grains will grind themselves down through collisions until they are small enough to be lost through PR drag, i.e., until $\tau_{\mathrm{PR}} \sim \tau_{\text {coll }}$. Following Sheret et al. (2004; see their Fig. 3) we note that radiation blowout does not operate efficiently for low-luminosity stars like HD 69830, particularly for nonidealized grains that are either porous or nonspherical (Li \& Greenberg 1998). Collisions followed by loss via PR drag are likely to dominate the grain distribution around HD 69830. Although the uncertainties on the HD 69830 timescales are large (at least a factor of 2 because of unknown grain size and orbital location), it is clear that small grains must be replenished on roughly the $<1000 \mathrm{yr}$ timescale or the emission associated them will disappear.

\subsubsection{An Enhanced Zodiacal Dust Cloud}

The probable source for the small grains seen toward HD 69830 is a debris cloud located within $1 \mathrm{AU}$ of the star that is replenished on timescales of $10^{3} \mathrm{yr}$. For comparison, our zodiacal cloud consists of relatively large grains $(10-100 \mu \mathrm{m})$ extending to $3 \mathrm{AU}$ and maintained in rough equilibrium, with occasional upward spikes, through asteroid collisions every few million years (Fixsen \& Dwek 2002; Dermott et al. 2002a, 2002b).

The mass in $0.25 \mu \mathrm{m}$ dust grains orbiting HD 69830 (§ 4.2), $4.6 \times 10^{-9} M_{\oplus}$, is destroyed in $\tau_{\text {coll }} \sim \tau_{\mathrm{PR}} \sim(0.5-1) \times 10^{3} \mathrm{yr}$ for a mass-loss rate of $(0.5-1) \times 10^{-11} M_{\oplus} \mathrm{yr}^{-1}$. This loss mechanism represents the endpoint for the small grain debris generated by the collisional cascade of larger bodies into smaller ones. If this mechanism operates continuously over $2 \mathrm{Gyr}$, then the total mass of solid material lost to the system over $2 \mathrm{Gyr}$ would be $>10^{-2} M_{\oplus}$, or more than 500 times the amount of material in own asteroid belt. Note that the estimate of solid material lost over time is also considerably greater than the present-day mass estimate, $(1-3) \times 10^{-3} M_{\oplus}$, inferred from integrating an $a^{-3.5}$ distribution up to either 10 or $100 \mathrm{~km}$ bodies $(\S 4.2)$. Either we are seeing the decaying remnant of a more massive disk, or the mass loss is episodic and the average massloss rate is not as great as present-day observations would suggest.

Another way to estimate the amount of material present is to examine the dust production rate. If, as noted above (Sheret et al. 2004), radiation blowout is not effective around HD 69830, then PR drag is the dominant loss for dust in HD 69830 and the number of emitting grains in the disk, $n_{\mathrm{gr}}$, is simply proportional to the square of the number of large bodies (comets or asteroids), $N_{c}$, that initiate the collisional cascade leading to these small grains: $n_{\mathrm{gr}} \propto N_{c}^{2}$ (Dominik \& Decin 2003). Presently, our zodiacal 
cloud has a fractional surface density around $10^{-7}$, but immediately after a major collision, the cloud would have a larger population of small grains given by the $n(a) \propto a^{-3.5}$ law appropriate to a collisional cascade (Dohnanyi 1969). The surface area of emitting material and the resulting optical depth of such a cloud is $\propto \int a^{2} a^{-3.5} d a \propto a^{-0.5}$. Extending the small grain cutoff from its present $10-0.25 \mu \mathrm{m}$ would temporally increase the emitting surface area by a factor of at least $\sim 6$ and probably more, since a collision would increase the amount of dust of all sizes. If we compare the cloud around HD 69830 with our solar system (enhanced to include short-lived $0.25 \mu \mathrm{m}$ grains for this calculation $)$, we need $\Sigma_{\text {frac }}($ HD 69830$) / \Sigma($ solar system $)=3 \times$ $10^{-4} / 6 \times 10^{-7}=500$ times more small grains orbiting HD 69830 than our zodiacal cloud would have immediately after a major collision.

Since, as argued above, $\Sigma_{\text {frac }} \propto N_{c}^{2}$ (Dominik \& Decin 2003), the number of large bodies required to replenish the dust would be $N_{c} \propto 500^{1 / 2}=22$ more than in our solar system. By this reasoning the total mass of a putative asteroid belt would be $0.3 \times 10^{-3} M_{\oplus}$, which compares favorably with the value of $1 \times 10^{-3} M_{\oplus}$ derived from integrating an $a^{-3.5}$ size distribution up to $10 \mathrm{~km}$. Although both mass estimates would increase if the upper size mass limit were increased to $100 \mathrm{~km}$, the values are considerably smaller than the $10^{-2} M_{\oplus}$ estimated by integrating the instantaneous mass-loss rate over the lifetime of the star. The simplest hypothesis is that the numbers are consistent within the uncertainties (particularly since the derived masses depend on the unconstrained upper end of the integral over the particle size distribution, $10-100 \mathrm{~km}$ ). If the difference between the instantaneous and long-term mass rates is significant, then we could be seeing a transient peak in surface area, possibly from a very recent collision, $<\tau_{\mathrm{PR}} \sim 10^{3} \mathrm{yr}$.

The exact details of an asteroid belt model will have to be established by a combination of improved modeling to constrain grain properties and location more precisely, as well as more realistic modeling of the dynamics. The simplest explanation for HD 69830's excess is an asteroid belt with much higher density located much closer to the star than in our solar system. A transient brightening with an excess of small grains in addition to the more massive asteroid system cannot be ruled out. Pursuing the analogy with our solar system suggests that an as yet unseen planet located outside $r_{\max }$ could truncate the disk and confine most of the dust to radii interior to the planet's orbit (Menou \& Tabachnik 2003). Such a planet could also enhance the lifetime of dust by trapping it into mean motion resonances (Kuchner \& Holman 2003).

\subsubsection{A Swarm of Comets as a Source of Small Grains}

An alternative to emission from small grains in an enhanced asteroid belt is emission from cometary ejecta. Levison et al. (2001) discuss possible mechanisms for stirring up the Kuiper Belt, including scattering of material into the inner solar system by the late formation of distant planets or the destabilization of Kuiper Belt objects due to sudden changes in the orbital parameters of one or more giant planets. Alternatively, some perturbing event might send a single large object located far from the star, i.e., a $1000 \mathrm{~km}$ sized object like Sedna (Brown et al. 2004), into the inner HD 69830 system where it becomes a "supercomet." We examine these two possibilities below.

What would it take to reproduce the emission from HD 69830 using cometary debris? Consider the $F_{\nu}(11.3 \mu \mathrm{m})=$ 7300 Jy flux density of Hale-Bopp measured in a 9."3 beam when Hale-Bopp was located at $r_{h}=1.1 \mathrm{AU}$ from the Sun and $\Delta_{\mathrm{HB}}=1.6 \mathrm{AU}$ from the Earth (Williams et al. 1997). If we scale this to Hale-Bopp's perihelion values of $r_{h}=0.9 \mathrm{AU}$ and $\Delta_{\mathrm{HB}}=1.5 \mathrm{AU}$ according to $r_{h}^{-4} \Delta^{-1}$ (Gehrz \& Ney 1992; Wooden et al. 1999) and to a radius of $20^{\prime \prime}$ (Hayward et al. 2000 ), then the total $11.3 \mu \mathrm{m}$ emission of this object would be $\sim 6 \times 10^{4} \mathrm{Jy}$. Placed at the $\Delta(\mathrm{HD} 69830)=12.6 \mathrm{pc}$ distance of HD 69830, it would take

$$
N_{\mathrm{HB}}=\frac{F_{\nu}(\mathrm{HD} 69830)}{F_{\nu}(\mathrm{HB})}\left[\frac{\Delta(\mathrm{HD} 69830)}{\Delta(\mathrm{HB})}\right]^{2} \sim 6 \times 10^{6}
$$

comets identical to Hale-Bopp as it appeared at 0.9 AU to reproduce the 0.013 Jy flux density measured by Spitzer at $11.3 \mu \mathrm{m}$. Reducing the periastron distance to $0.5 \mathrm{AU}$ consistent with the $\sim 400 \mathrm{~K}$ temperature determined in the modeling would increase the brightness of the cometary material and thus decrease their required number to $\sim 5 \times 10^{5}$ Hale-Bopp-like comets.

Consider a steady stream of comets on $P=10^{3} \mathrm{yr}$ orbits arriving at the inner HD 69830 system at a rate of $\eta$ comets $\mathrm{yr}^{-1}$. If each comet is bright while it is within $3 \mathrm{AU}$ for a time $t_{3 \mathrm{AU}} \sim 1 \mathrm{yr}$ and makes $N_{\text {pass }} \sim 100$ active passes through the inner HD 69830 system (Grün et al. 2001; Wooden et al. 2000), then in steady state $N_{\mathrm{HB}}=\eta t_{3} \mathrm{AU}$ comets contribute to the observed excess at any one time; $N_{\text {active }}=\eta P$ contribute to the excess whenever they come close to the star, and $N_{\text {total }}=$ $\eta$ lifetime $/ N_{\text {pass }}$ comets contribute to the excess during the putative $10^{7} \mathrm{yr}$ lifetime of the excess. If, as argued above, we need $5 \times 10^{5}$ Hale-Bopp-like comets at any instant, then $\eta=$ $5 \times 10^{5}$ comets $\mathrm{yr}^{-1}$. With these numbers, $N_{\text {active }}=5 \times 10^{8}$ and $N_{\text {total }}=5 \times 10^{10}$ over the duration of this excess. With a total mass for Hale-Bopp of $\sim 1 \times 10^{20} \mathrm{~g}$, assuming a density of $1.5 \mathrm{~g} \mathrm{~cm}^{-3}$ for a mixture of grain and ice and a radius of $\sim 25 \mathrm{~km}$ (Weaver \& Lamy 1997), the total mass in comets entering the inner HD 69830 system during this $10^{7}$ yr period would be $\sim 900 M_{\oplus}$, unreasonably large for any residual Kuiper Belt. Even if more normal, smaller comets were adopted, e.g., a factor of 10 smaller than Hale Bopp, the total mass would still require $\sim 90 M_{\oplus}$ in comets, which still seems large given the $70 \mu \mathrm{m}$ limits on a distant Kuiper Belt. While the uncertainty in this simple model is large, the total mass of comets required to maintain the hot material argues against the cometary hypothesis.

\subsubsection{A Supercomet as a Source of Small Grains}

An alternative to a swarm of comets is a single giant comet scattered into the inner HD 69830 system, perhaps an object like Sedna with a radius of $1000 \mathrm{~km}$ and composed of ice and rock. Such an object would have a surface area $(1000 / 25)^{2}=$ 1600 times that of Hale-Bopp and in a low-eccentricity, $0.5 \mathrm{AU}$ orbit would evolve gas and small dust grains at a rate $d M(t) / d t=$ 1600 times greater than Hale-Bopp. In an idealized picture, this material would build up over $\tau_{\mathrm{PR}}$, would last until the entire comet evaporated in a time $\tau_{\text {evap }}$, and then decline in $\tau_{\text {PR }}$. During $\tau_{\text {evap }}$ the average amount of material would be equivalent to $d M(t) / d t\left(\tau_{\mathrm{PR}}\right) \sim 1.6 \times 10^{6}$ Hale-Bopp-like comets, which agrees reasonably well with the amount of emitting material estimated in $\S$ 4.4.3.

We can estimate the evaporation time by noting that the mass-loss rate is proportional to the surface area of the comet: $d M(t) / d t=4 \pi \Phi R(t)^{2}$, where $\Phi$ is the flux of material emitted in $\mathrm{g} \mathrm{cm}^{-2} \mathrm{~s}^{-1}$ and $R(t)$ is the radius as a function of time. Then, with $R(t)=[3 M(t) / 4 \pi \rho]^{1 / 3}$ and $M(0)=4 / 3 \pi \rho R(0)^{3}$, we get

$$
M(t)=\frac{4 \pi[R(0) \rho-\Phi t]^{3}}{3 \rho^{2}}
$$


and $\tau_{\text {evap }}=R(0) \rho / \Phi$. At perihelion, Hale-Bopp gave up some 200 metric tons s ${ }^{-1}$ in gas and small grains (Lisse et al. 1999) and possibly as much as 2000 metric tons s ${ }^{-1}$ in larger grains detected at submillimeter wavelengths (Jewitt \& Matthews 1999). For Hale-Bopp with an assumed $25 \mathrm{~km}$ radius and a mass-loss rate of 200 tons s $\mathrm{s}^{-1}$ for small grains, $\Phi=76 \mathrm{~g} \mathrm{~cm}^{-2}$ $\mathrm{s}^{-1}$. An escape velocity from a Sedna-like object is about $0.5 \mathrm{~km}$ $\mathrm{s}^{-1}$, half that of Pluto, and comparable to the thermal velocity of $\mathrm{H}_{2} \mathrm{O}$ at $300 \mathrm{~K}$. Small grains entrained with the gas could escape the nucleus, while larger grains would probably fall back under the self-gravity of the object. Therefore, we adopt the lower, small grain mass-loss estimate for this putative supercomet. It is worth noting that this grain segregation mechanism would account naturally for the lack of large grains with their associated long-wavelength emission.

A $1000 \mathrm{~km}$ sized comet trapped in a low-eccentricity orbit (making $\Phi$ roughly constant) would have an evaporation lifetime of $\tau_{\text {evap }}=2 \mathrm{Myr}$ and could provide material over a long enough time to have a reasonable chance of being observed by Spitzer. Effects of self-gravity and ice-rock differentiation would reduce the flux of evolved material but would extend the duration of the outburst. This scenario would be less attractive if the supercomet were on a highly eccentric orbit, spending much of its time away from the star. The requirement for the supercomet to travel in a low-eccentricity orbit close to the star suggests the presence of a planet located close to the star to effect its capture.

Finally, we note that the rupture of a single Sedna-like object in the inner HD 69830 system could release a swarm of $10^{5}-10^{6}$ smaller, $10-25 \mathrm{~km}$ bodies, which in turn could produce a large number of small grains as envisioned in the preceding section. Since the small grains would last only $\tau_{\mathrm{PR}}$, a significant fraction of the swarm would have to survive for a few million years to replenish the small grains and thus to make the outburst last long enough for us to have a chance to see it.

In either case, the Sedna-like object could have been dislodged from a stable, more distant orbit by a planet in the HD 69830 system or by the effects of a passing star. The Sedna-like object might even have been captured from the passing star itself (Kenyon \& Bromley 2004), although the space motion of HD 69830 (Eggen 1998) indicates no nearby approaches to any known star within the past $20 \mathrm{Myr}$ (V. Makarov 2005, private communication).

\subsubsection{An Observational Test for the Origin of the HD 69830 Excess}

There is a powerful observational test that could decide between the two models. The cometary scenario would bring a great deal of volatile material into the inner HD 69830 system. In Hale-Bopp, species such as $\mathrm{CO}, \mathrm{H}_{2} \mathrm{O}, \mathrm{HCN}$, and oxygen were detected at wavelengths from the millimeter to the ultraviolet. Sensitive spectroscopy of HD 69830 might reveal evidence for volatiles seen either in emission or in absorption against the star, similar to what has been detected toward $\beta$ Pic in the visible (Lagrange-Henri et al. 1992; Petterson \& Tobin 1999) and in the UV (Vidal-Madjar et al. 1994; Roberge et al. 2000), and toward HD 100546 (Vieira et al. 1999; Bouwman et al. 2003). While examination of the IUE archive spectrum of HD 69830 (Hufnagel \& Smith 1994) shows no features attributable to gas in a shell around the star as is seen in $\beta$ Pic, e.g., excess Mg II absorption at 279.5 and $280 \mathrm{~nm}$, more careful searches for volatile material are clearly warranted. Detection of such volatiles would strongly support the cometary hypothesis and represent a milestone in our understanding of how organic molecules are transported from the cold outer solar system into the region of the terrestrial planets.

\section{THE DETECTABILITY OF PLANETS IN THE HD 69830 SYSTEM}

Finally, we note that the intense diffuse emission toward this star makes it unlikely that future astronomical telescopes will ever detect directly the light from terrestrial planets in the HD 69830 system. For telescopes such as the Terrestrial Planet Finder (TPF; Beichman 2003), the photon noise at either visible or mid-IR wavelengths associated with a target's exozodiacal cloud begins to dominate other noise sources at levels exceeding 10 times the brightness of our own zodiacal cloud. Unfortunately, the HD 69830 cloud, being more than 1000 times brighter than our cloud, will hide planets like our own from view.

From the standpoint of TPF it is useful to note that the limiting IRS sensitivity described herein will allow us to detect or set limits to small grains located in the "habitable zones" at least a factor of 10 fainter than that around HD 69830. A focused program of IRS observations should reach $\sim 100$ times the level of our zodiacal cloud and thus provide a good initial filter for $T P F$ target selection.

\section{CONCLUSION}

We have identified an excess of emission between 8 and $35 \mu \mathrm{m}$ toward the nearby K0 V star HD 69830. The IRS spectrum of the excess reveals prominent features due to crystalline silicates such as forsterite, which must be present in small, submicron dust grains for the features to be so prominent. The lack of longer wavelength emission and the ratios of various spectral features suggest that most of this material has a temperature in excess of $400 \mathrm{~K}$ and must be located within $1 \mathrm{AU}$ of the star. An excess of this type (strong at $25 \mu \mathrm{m}$ but weak at $70 \mu \mathrm{m}$ ) is unique among the sample of 84 main-sequence stars examined by Beichman et al. (2005) and in other IRAS/ISO samples, implying a short-lived event or a low duty cycle process.

Estimates of the mass in an enhanced asteroid belt needed to replenish the small dust grains seen by Spitzer are quite uncertain: (1) 64 times the amount in our solar system based on an extrapolation of the emitting surface area of small grains up to $10 \mathrm{~km}$ bodies or (2) 22 times the amount in our solar system based on dust production in asteroid-asteroid collisions. If the mass-loss rate inferred from the present day excess were constant over $2 \mathrm{Gyr}$, the system would have lost at least 500 times the amount of asteroidal material in our solar system. A massive, steady state asteroid belt is a likely explanation, but a transient brightening due to a recent collision creating a large number of small grains cannot be ruled out.

Alternatively, small grains could arise from a single, Sednasized supercomet recently captured in the inner HD 69830 system and "evaporating" over a few million years or breaking apart into smaller objects. This supercomet would have to be trapped in a low-eccentricity orbit around $1 \mathrm{AU}$ from the star. Spectroscopic follow-up to search for volatiles associated with comets would help distinguish between these two hypotheses.

We would like to thank Geoff Marcy, Debra Fischer, and John Stauffer for valuable discussions on the properties of HD 69830. Sergio Fajardo-Acosta provided programs we used for accessing the Kurucz models. We acknowledge useful discussions with Doug Lin on the relative merits of asteroid and 
Kuiper Belts as a source of the excess, Valeri Markarov on the recent trajectory of HD 69830, and Bill Reach on the physical properties of comet trails. The anonymous referee provided a number of very useful remarks about the origin of dust in an asteroid belt and the relevant timescales. This research made use of the IRAS, 2MASS, and Hipparcos catalogs (ESA 1997), as well as the SIMBAD database and the VizieR tool operated by CDS, Strasbourg, France. The Spitzer Space Telescope is operated by the Jet Propulsion Laboratory, California Institute of Technology, under NASA contract 1407. Development of MIPS was funded by NASA through the Jet Propulsion Laboratory, subcontract 960785 . Some of the research described in this publication was carried out at the Jet Propulsion Laboratory, California Institute of Technology, under a contract with the National Aeronautics and Space Administration. Finally, we remember with great sadness the efforts of NRC postdoctoral fellow Elizabeth Holmes, who worked intensively on this project before her untimely death in 2004 March.
Aumann, H. H., \& Good, J. C. 1990, ApJ, 350, 408

Aumann, H. H., et al. 1984, ApJ, 278, L23

Backman, D. E. 1998, in Exozodiacal Dust Workshop, ed. D. E. Backman et al. (Moffett Field: NASA), 107

Backman, D. E., \& Paresce, F. 1993, in Protostars \& Planets III, ed. E. H. Levy \& J. I. Lunine (Tucson: Univ. Arizona Press), 1253

Beichman, C. 2003, in ASP Conf. Ser. 291, Hubble's Science Legacy: Future Optical/Ultraviolet Astronomy from Space, ed. K. R. Sembach et al. (San Francisco: ASP), 101

Beichman, C., Chester, T., Gillet, F. C., Low, F. J., Matthews, K., \& Neugebauer, G. 1990, AJ, 99, 1569

Beichman, C., et al. 2005, ApJ, 622, 1160

Bidstrup, P. R., Michelsen, R., Andersen, A. C., \& Haack, H. 2005, A\&A, in press

Bouwman, J., deKoter, A., Dominik, C., \& Waters, L. B. F. M. 2003, A\&A, 401, 577

Bowey, J. E., Lee, P. C., Tucker, C., Hofmeister, A. M., Ade, P. A. R., \& Barlow, M. J. 2001, MNRAS, 325, 886

Bradley, J. 2003, in Astromineralogy, ed. T. K. Henning (Berlin: Springer), 217

Brown, M. E., Trujillo, C. A., Rabinowitz, D., Stansberry, J., Bertoldi, F., \&

Koresko, C. D. 2004, AAS/DPS Meeting, 36, 03.01

Burns, J., Lamo, P. L., \& Soter, S. 1979, Icarus, 40, 1

Castelli, F., \& Kurucz, R. L. 2003, in IAU Symp. 210, Modelling of Stellar Atmospheres, ed. N. E. Piskunov, W. W. Weiss, \& D. F. Gray (San Francisco: ASP), A20

Cayrel de Strobel, G., Soubiran, C., \& Ralite, N. 2001, A\&A, 373, 159

Cox, A. N., ed. 2000, Allen's Astrophysical Quantities (New York: Springer)

Crovisier, J., et al. 1996, A\&A, 315, L385 1997, Science, 275,1904

Decin, L., et al. 2003, A\&A, 400, 679

Dent, W. R. F., Walker, H. J., Holland, W. S., \& Greaves, J. S. 2000, MNRAS, 314,702

Dermott, S. F., Durda, D. D., Grogan, K., \& Kehoe, T. J. J. 2002a, in Asteroids III, ed. W. F. Bottke, Jr. et al. (Tucson: Univ. Arizona Press), 423

Dermott, S. F., Kehoe, T. J. J., Durda, D. D., Grogan, K., \& Nesvorný, D. 2002, in Proc. Asteroids, Comets, Meteors: ACM 2002b, ed. B. Warmbein (ESA SP-500; Noordwijk: ESA), 319

Dijkstra, C., Waters, L. B. F. M., Kemper, F., Min, M., Matsuura, M., Zijlstra, A., de Koter, A., \& Dominik, C. 2003, A\&A, 399, 1037

Dohnanyi, J. S. 1969, J. Geophys. Res., 74, 2431

Dominik, C., \& Decin, G. 2003, ApJ, 598, 626

Draine, B. T., \& Lee, H. M. 1984, ApJ, 285, 89

Eggen, O. 1998, AJ, 115, 2397

ESA. 1997, The Hipparcos Catalogue (VizieR Online Data Catalog, 1239)

Fajardo-Acosta, S., Beichman, C., \& Cutri, R. 2000, ApJ, 538, L155

Fajardo-Acosta, S., Telesco, C. M., \& Knacke, R. F. 1993, ApJ, 417, L33

Fixsen, D. J., \& Dwek, E. 2002, ApJ, 578, 1009

Gehrz, R. D., \& Ney, E. P. 1992, Icarus, 100, 162

Gillett, F. C. 1986, in Light on Dark Matter, ed. F. P. Israel (Dordrecht: Reidel), 61

Gordon, K. D., et al. 2005, Proc. SPIE, in press

Grady, C. A., Sitko, M. L., Bjorkman, K. S., Perez, M. R., Lynch, D. K., Russell, R. W., \& Hanner, M. S. 1997, ApJ, 483, 449

Greaves, J. S., Wyatt, M. C., Holland, W. S., \& Dent, W. R. F. 2004, MNRAS, 351, L54

Greaves, J. S., et al. 1998, ApJ, 506, L133

Grün, E., et al. 2001, A\&A, 377, 1098

Habing, H. J., et al. 2001, A\&A, 365, 545

\section{EFERENCES}

Hayward, T. L., Hanner, M. S., \& Sekanina, Z. 2000, ApJ, 538, 428

Heap, S. R., et al. 2000, ApJ, 539, 435

Henning, T. 1998, Chem. Soc. Rev., 27, 315

Holland, W. S., et al. 1998, Nature, 392, 788

Houck, J. R., et al. 2004, ApJS, 154, 18

Hufnagel, B., \& Smith, G. H. 1994, PASP, 106, 1068

Jäger, C., Molster, F. J., Dorschner, J., Henning, Th., Mutschke, H., \& Waters, L. B. F. M. 1998, A\&A, 339, 904

Jewitt, D., \& Matthews, H. 1999, AJ, 117, 1056

Jura, M., et al. 2004, ApJS, 154, 453

Kelsall, T., et al. 1998, ApJ, 508, 44

Kenyon, S. J., \& Bromley, B. C. 2004, Nature, 432, 598

Koike, C., Shibai, H., \& Tuchiyama, A. 1993, MNRAS, 264, 654

Krasinsky, G. A., Pitjeva, E. V., Vasilyev, M. V., \& Yagudina, E. I. 2002, Icarus, 158,98

Kuchner, M. J., \& Holman, M. J. 2003, ApJ, 588, 1110

Kurucz, R. L. 1992, in IAU Symp. 149, The Stellar Populations of Galaxies, ed. B. Barbuy \& A. Renzini (Dordrecht: Kluwer), 225

Lagrange-Henri, A. M., Gosset, E., Beust, H., Ferlet, R., \& Vidal-Madjar, A. 1992, A\&A, 264, 637

Laureijs, R. J., et al. 2002, A\&A, 387, 285

Levison, H. F., Dones, L., Chapman, C. R., Starn, A., Duncan, M. J., \& Zahnle, K. 2001, Icarus, 151, 286

Li, A., \& Greenberg, J. M. 1998, ApJ, 498, L83

Lisse, C. M., et al. 1999, Earth Moon Planets, 78, 251

Malfait, K., Waelkens, C., Waters, L. B. F. M., Vandenbussche, B., Huygen, E., $\&$ de Graauw, M. S. 1998, A\&A, 332, L25

Mannings, V., \& Barlow, M. J. 1998, ApJ, 497, 330

Meeus, G., Waters, L. B. F. M., Bouwman, J., van den Ancker, M. E., Waelkens, C., \& Malfait, K. 2001, A\&A, 365, 476

Menou, K., \& Tabachnik, S. 2003, ApJ, 583, 473

Moshir, M., et al. 1990, BAAS, 22, 1325

Mukai, T., \& Koike, C. 1990, Icarus, 87, 180

Petterson, O. K. L., \& Tobin, W. 1999, MNRAS, 304, 733

Reach, W. T., Morris, P., Boulanger, F., \& Okumura, K. 2003, Icarus, 164, 384

Roberge, A., Feldman, P. D., Lagrange, A. M., Vidal-Madjar, A., Ferlet, R., Jolly, A., Lemaire, J. L., \& Rostas, F. 2000, ApJ, 538, 904

Sheret, I., Dent, W. R. F., \& Wyatt, M. C. 2004, MNRAS, 348, 1282

Skiff, B. A. 2003, General Catalogue of Stellar Spectral Classifications (Flagstaff: Lowell Obs.), http://cdsweb.u-strasbg.fr/viz-bin/Cat?III/233A

Smith, B. A., \& Terrile, R. J. 1984, Science, 226, 1421

Song, I., Caillault, J.-P., Barrado y Navascués, D., Stauffer, J. R., \& Randich, S. 2000, ApJ, 533, L41

Stapelfeldt, K. R., et al. 2004, ApJS, 154, 458

Stern, A. S. 1996, A\&A, 310, 999

Telesco, C. M., \& Knacke, R. F. 1991, ApJ, 372, L29

Vidal-Madjar, A., et al. 1994, A\&A, 290, 245

Vieira, S. L. A., Pogodin, M. A., \& Franco, G. A. P. 1999, A\&A, 345, 559

Weaver, H. A., \& Lamy, P. L. 1997, Earth Moon Planets, 79, 17

Weingartner, J. C., \& Draine, B. T. 2001, ApJ, 548, 296

Williams, D. M., et al. 1997, ApJ, 489, L91

Wooden, D. H., Butner, H. M., Harker, D. E., \& Woodward, C. E. 2000, Icarus, 143,126

Wooden, D. H., Harker, D. E., Woodward, C. E., Butner, H. M., Chiyoe, K., Witteborn, F. C., \& McMurtry, C. W. 1999, ApJ, 517, 1034

Wright, J. T., Marcy, G. W., Butler, R. P., \& Vogt, S. S. 2004, ApJS, 152, 261

Yoneda, S., Simon, S. B., Sylvester, P. J., Hsu, A., \& Grossman, L. 1993, Meteoritics, 28, 465 